Joyzukey Armendáriz Hernández. Es licenciada en Lengua Inglesa y maestra en Educación Superior por la Facultad de Filosofía y Letras de la Universidad Autónoma de Chihuahua, institución en la cual colabora como docente de asignaturas de Literatura Inglesa Medieval, Romántica e Isabelina. Ha publicado artículos referentes a la narrativa de J.R.R. Tolkien en Cinzontle, Metamorfosis, Humanitas, y Literal Ink, y un análisis sobre educación superior en la revista indexada NAER-Journal of New Approaches in Educational Research. Actualmente, es becaria CONACYT en el Doctorado en Filosofía con Acentuaciones en Estudios de la Cultura en la Universidad Autónoma de Nuevo León con un proyecto de tesis relacionado al texto inglés medieval y la narrativa de J.R.R. Tolkien. El texto a incluir es parte del proyecto de investigación de la tesis "Aproximaciones a la espiritualidad en la narrativa de J.R.R. Tolkien”.

Víctor Barrera Enderle. Es investigador de tiempo completo en la Facultad de Filosofía y Letras de la Universidad Autónoma de Nuevo León (UANL) y director de la revista Armas y Letras. Es licenciado en Letras Españolas por esta casa de estudios, y maestro en Literatura con Mención en Teoría Literaria y doctor en Literatura Hispanoamericana por la Universidad de Chile. Ha publicado artículos en revistas especializadas y capítulos de libros en proyectos colectivos. Su obra académica y ensayística le ha valido el reconocimiento del Instituto Iberoamericano de Berlín, del que fue becario de investigación en 2004, así como el premio del Certamen Nacional de Literatura Alfonso Reyes 2006.

Jorge Alan Flores Flores. Posee la doble licenciatura en Derecho y en Filosofía, Doble maestría en Humanidades y en Derechos Humanos, y es Doctor en Filosofía con Acentuación en Estudios de la Cultura, por la Universidad Autónoma de Nuevo León, (UANL), titulado con mención Magna Cum Laude. Es también especialista en Estudios de la Frontera México-EEUU, y tiene Diplomados en Gobierno y Dirección pública, y Economía. Ha sido becario en programas como el ICHICULT y CONACYT. También ha fungido como Director Editorial de la revista Metamorfosis ISSN: 2007-6525 FFyL de la UACH.

Historial editorial

Recepción: 20 de enero de 2021

Revisión: 28 de enero de 2021

Aceptación: 13 de abril de 2021 Publicación: 28 de junio de 2021 


\title{
Presencia de la espiritualidad en el texto inglés medieval (siglos VII-XII)
}

\author{
Presence of spirituality in medieval \\ English text (7th-12th centuries)
}

\section{Presença da espiritualidade no texto medieval em inglês (séculos VII-XII)}

\author{
Joyzukey Armendáriz Hernández / Víctor Barrera Enderle / \\ Jorge Alan Flores Flores
}

Universidad Autónoma de Nuevo León/Universidad Autónoma de Nuevo León/ Universidad Autónoma de Chihuahua

joy_armendariz@yahoo.com/vicbarrera@hotmail.com/jorgealanf@gmail.com

\section{Resumen}

El presente artículo observará los textos literarios icónicos de la literatura medieval anglosajona en su traducción del inglés antiguo o anglosajón al inglés moderno, y describirá el concepto de espiritualidad en el contexto medieval para evaluar de qué manera esta definición se aplica a diversos elementos del imaginario narrativo. No se busca evaluar la traducción, empresa que podrá iniciarse en un periodo posterior. No obstante, se prefieren las traducciones que enfatizan el significado y uso de imágenes en el texto a la preservación del ritmo literario. Ejemplo de lo anterior es la traducción del texto Beowulf, realizado por el poeta irlandés Seamus Heaney. Se realizará entonces una evaluación de la literatura de la época Anglo-sajona en Britania incluyendo los textos The Seafarer, The destruction of Anglo-saxon temples y Caedmon de Bede, y finalmente Beowulf. Los hechos políticos, sociales e intelectuales se considerarán para análisis a través de las selecciones de los textos. Las características poéticas se analizarán e interpretarán para sustentar sobre la temática principal de espiritualidad. El análisis tiene un enfoque exploratorio y no pretende ser exhaustivo debido a los lineamientos pertinentes.

Palabras clave: Espiritualidad, Imaginario medieval, Beowulf, Literatura medieval anglosajona. 


\section{Abstract}

The article will observe iconic literary texts from the Anglo-Saxon medieval time translated from old English into modern English to describe the concept of spirituality in this medieval context in order to assess the way this idea is applicable to the different examples of narrative. It is not the purpose of this article to assess the effectiveness of the translations, such research might be conducted at a later time. Nonetheless, translations that rely mostly on meaning and the use of images are preferred, rather than those that focus solely on rhythm. An example of this is the translation of Beowulf by irish poet Seamus Heaney. An assessment of the literary examples of the Anglosaxon time will be conducted, which includes the following texts: The Seafarer, The destruction of Anglo-saxon temples and Bede's Caedmon, and finally Beowulf. Political, social, and intellectual events will be considered for this analysis of the texts. Poetic characteristics will be analyzed and interpreted in order to support the main idea of spirituality. This analysis in only exploratory and does not intend to be thorough given the circumscription.

\section{Keywords: Spirituality, Medieval Imaginary, Beowulf, Anglo-Saxon medieval literature.}

\section{RESUMO}

O artigo observará textos literários icônicos da época medieval anglo-saxônica traduzidos do inglês antigo para o inglês moderno para descrever o conceito de espiritualidade nesse contexto medieval, a fim de avaliar como essa ideia é aplicável aos diferentes exemplos de narrativa. Não é o objetivo deste artigo avaliar a eficácia das traduções, tais pesquisas podem ser conduzidas em um momento posterior. No entanto, traduções que se baseiam principalmente no significado e no uso de imagens são preferidas, em vez daquelas que se concentram apenas no ritmo. Um exemplo disso é a tradução de Beowulf pelo poeta irlandês Seamus Heaney. Será realizada uma avaliação dos exemplos literários da época Anglosaxon, que inclui os seguintes textos: The Seafarer, A destruição dos templos Anglo-saxões y Caedmon de Bede, e finalmente Beowulf. Eventos políticos, sociais e intelectuais serão considerados para esta análise dos textos. Características poéticas serão analisadas e interpretadas de forma a fundamentar a ideia central de espiritualidade. Esta análise é apenas exploratória e não pretende ser exaustiva dada a circunscrição.

\section{Palavras-chave: Espiritualidade, imaginário medieval, Beowulf, liter- atura medieval anglo-saxã.}




\section{INTRODUCCIÓN}

El estudio y análisis de la literatura medieval está íntimamente ligado al pensamiento cultural de la época. Separar uno de otro sería limitar el enfoque que inicialmente el texto buscaba obtener. De la misma manera, entender el desarrollo histórico sin apoyo de los textos literarios medievales sería minimizar la crónica histórica y negar el desarrollo literario.

En el caso particular de la literatura inglesa, se observa un enramado de múltiples creencias que se fueron embonando para dar lugar a los diversos ejemplos literarios y de comportamiento que podemos disfrutar hoy.

En combinación con la heroica presencia del guerrero, la fe y providencia mezclaban elementos paganos y cristianos en un despliegue de creencias difíciles de definir. Los Romanos no tenían la intención de eliminar el paganismo, sino aplicar el cristianismo a través de las mismas creencias culturales, para que de esta manera, se instaurara la nueva religión, y eventualmente sustituyera las creencias antiguas. Clemente de Alejandría indica que incluso algunos filósofos como Platón habían encontrado a Dios en su propio camino, aunque éste se alejara del ideal cristiano de la plenitud del conocimiento a través del Logos, o Cristo. Por lo tanto, sus obras buscan reforzar el proceso de maduración del creyente, para encontrar el camino de la verdad, bajo la enseñanza del maestro por excelencia: Cristo. Se menciona, presuntamente atribuido a Clemente, en la compilación de Reconocimientos, Libro 10 (1867): "Éstos (dioses griegos) de quienes se habla, no solo como hombres que han muerto, sino como impíos que han sido castigados por sus crímenes; y sin embargo, son adorados por hombres necios como dioses..."

La adjudicación o asimilación de entidades divinas en la antigua Roma se dio de forma sincrética o de adopción. Entre mayores territorios se conquistaban, más aumentaba el listado de deidades, sin embargo, esto se hacia bajo el principio de asimilación a los dioses romanos que poseían características similares a la nueva deidad. Es así como la triada capitolina con Júpiter a la cabeza, seguido de Juno y Minerva asemeja de alguna manera la postrera trinidad cristiana.

De esta manera, existe una mezcla de ambas corrientes que invariablemente fomentaba confusión, especialmente entre reyes y líderes, centros de esas mismas crónicas.

Es importante entonces notar que las crónicas que evolucionaron de la oralidad tradicional, esas mismas que ensalzaban al rey

1 El presente texto es un capítulo del proyecto de investigación de tesis doctoral. 
guerrero, héroe valiente, líder incansable, hablan de esta dicotomía cultural. La literatura solo despliega los matices diversos de la multiplicidad de ideas espirituales, no busca explicarlos, sin embargo, y como se ha notado en una variedad de estudios críticos, hay una inclinación obvia a la aceptación del cristianismo.

Gracias al cristianismo, la visión de la vida se expande del modelo elegiaco Anglo-Sajón en el que el destino ya no solo es muerte sino vida eterna. También la cultura se vuelve accesible gracias al trabajo de los monjes. Sin embargo, es importante notar que algunos ejemplos de literatura medieval inglesa están manejados de manera irresponsable, ya que algunos monjes, presuntamente, se tomaban libertades creativas en pro de la supervivencia de sus creencias ; esto podría ejemplificar en los párrafos iniciales y finales de los poemas The Wanderer y The Seafarer encontrados en el códice de Exeter. Estos párrafos difieren con el tono elegiaco Anglo-sajón que enfatizaba la fama y el destino final de la muerte como el camino a seguir por el guerrero. Por el contrario, estos comentarios asemejan una plegaria visiblemente cristiana, al hacer mención del Padre Celestial. Y aún así, los monasterios se consideraron durante este tiempo medieval los centros intelectuales en donde se compartía el conocimiento más elevado.

La literatura Inglesa Medieval se desarrolló sobre la idea del héroe épico. La búsqueda de aventura y la destreza mostrada, elevaba a este hombre poco común al lugar privilegiado de la memoria colectiva. Nunca moriría si el pueblo continuaba relatando sus aventuras.

La teoría de la Literatura debe ser atemporal, para ofrecer una explicación del atractivo de los textos medievales en pleno siglo XXI. Debe explorar los valores que trascienden no solo a la persona, sino a la época. ¿Cuáles elementos enfatizan la trascendencia del Yo egocéntrico, y rescatan el Yo Épico que se inmortaliza?

Los textos medievales ingleses se escribieron en un contexto religioso, y es este mismo el que enfatiza la idea de desechar el yo, y permitir que la esencia heroica permanezca para instruir a generaciones posteriores. Aunque no solo es religión, el rol de la iglesia es innegable. La pertenencia se podría dar de múltiples maneras, como la pertenencia a un líder guerrero. Sin embargo, la pertenencia que aceptaba mayor número de adeptos era la eclesiástica. En el contexto religioso, esos individuos que posiblemente no tenían cabida en ningún otro grupo, finalmente encontraban una definición del Ser.

Entonces, para adentrarnos en el tema en cuestión, resumiremos que los textos a explorar se incluyen en los principales manuscritos medievales anglosajones. El manuscrito Cotton Vitellius, encua- 
dernado en el siglo XVII incluye la obra de Beowulf, que data previo al siglo IX. En segundo lugar se encuentra el manuscrito Junius, que incluye un texto del monje Caedmon, historia contada a través del relato del Venerable Bede. El códice de Exeter que incluye los poemas the Wanderer $y$ the Seafarer. Las versiones de las baladas incluidas corresponden a traducciones directas de inglés antiguo realizadas por el profesor James J. Whilhelm (1971), y la traducción por el profesor Nevill Coghill (1951) del prólogo de Los Cuentos de Canterbury escrito por Geoffrey Chaucer.

\section{LA ESPIRITUALIDAD COMO ENFOQUE}

La espiritualidad se conecta con la profundidad, la cultura, la serenidad, el sentido de pertenencia, la bondad. Espiritualidad se asocia también a la religiosidad, a la pertenecía a un culto. Por lo tanto, al escuchar el término espiritualidad, el oyente quisiera desasociarse dependiendo de la postura en la que considera su fe.

Según el teólogo y filósofo catalán Francesc Torralba, se pueden considerar 3 ámbitos en el contexto de la espiritualidad, el primero lleva el mismo nombre y se refiere a la trascendencia o a la voluntad de conocer los misterios. El segundo es la religiosidad en la que se reconoce un vinculo, una alteridad, que produce un cierto dialogo de petición, gratitud, o temor y temblor. En esto, hay una relación con algo o alguien que no soy yo. El tercero se denomina la confesionabilidad, que es la pertenencia a un entorno específico con textos sagrados y en el marco de una tradición con características históricas. El ser humano puede experimentar la espiritualidad ya presente en sí mismo, pero enfocada a alguno de estos tres ámbitos.

La espiritualidad según Astin (p 34) divide la percepción objetiva de la realidad, el actuar objetivo, a la cuestión subjetiva que el ser humano percibe solamente de manera interna, esto es, ideas sobre quiénes somos, hacia dónde vamos, cuál es nuestro propósito, cuál es el significado de la vida y nuestro trabajo, pero también la manera en la que entendemos otras cuestiones, de igual manera subjetivas, como la intuición, la inspiración, los misterios, el misticismo. Esto nos lleva a un sentido de asociación con otros seres que posiblemente también compartan estas ideas subjetivas. El enfoque de este autor esta dirigido a la transformación del currículo escolar, esto bajo la noción de que la estructuración tradicional de los temas en la educación está siempre fundamentada en elementos objetivos, y que las habilidades espirituales, también denominadas por él como afectivas, se han dejado de lado, y así se ha limitado muchísimo la indagación del ser. Astin indica que 
la conexión interpersonal está centrada en estos aspectos afectivos, y que la falta de atención a tales elementos (por parte del estudiante), o la falta de un currículo que incluya elementos afectivos y místicos, no permitirá un desarrollo integral en el cual el alumno, individuo, se desenvuelve de manera autentica, y por lo tanto, vivirá fragmentado, sin encontrar propósito o relevancia en lo que está persiguiendo.

También Grosh (p. 229) indica que la espiritualidad debe ser un proceso conectado con la educación, por lo tanto, no es un proceso innato, sino está fundamentado con el desarrollo de una conciencia activa. Grosh busca rescatar la importancia del concepto de espiritualidad a través de un análisis filosófico que se ha ido desgastando a través de los tiempos. Su argumento central yace en el término griego Paidea, o educación, cuyo fundamento es la cultivación de la humanidad y el refinamiento de la cultura. Esto solo se puede alcanzar si se presta mayor atención a la espiritualidad. Según la directriz de la inscripción en el templo de Delfos, Gnoti Seuton, el conocimiento del ser debe ser el propósito principal de la conducta humana, y mediante este proceso, se observa un doble rol, primero el ser como un representante de la raza humana, y segundo, el ser como individuo o entidad particular. Así parte de un proceso doble que define como la enseñanza de la espiritualidad, del concepto y sus prácticas asociadas, y el segundo es la educación a través de la espiritualidad en el que el proceso de la enseñanza está guiado por las prácticas que regulan una vida espiritual. Por lo tanto, se debe definir primeramente la idea de espiritualidad que gobernará el estudio, y después, definir las prácticas que bajo tal precepto se consideran espirituales, y cuál es el propósito de tales prácticas.

Históricamente, la espiritualidad había sido regida por la teoría moral Aristotélica, en la que se indican virtudes o cualidades deseadas, y los vicios que ocurren en caso de ausencia de tales virtudes. Esto guiaba al ser humano a cumplir su propósito, telos, a través del fortalecimiento de estas virtudes para convertirse en una persona, un ciudadano, de buena naturaleza. Sin embargo, después de Kant y el racionalismo, todo se centra en el raciocinio humano, a través del cual se construye una asociación de principios abstractos que justifican una moralidad según lo que el individuo considere correcto. Lo anterior también incluye, según Grosh, a Kierkegaard y su concepto de elección, como habilidad del libre albedrío, y esto propone un cambio de la moralidad observada a través del tiempo y de la perspectiva filosófica en la que ya se incluyen otros elementos como los placeres, el análisis de lo que es bueno o malo, la ética, etc. Como se puede observar, existe una desintegración de lo que podría ser considerada una teoría moral, 
lo cual fundamentaría el concepto general de espiritualidad. Debido a este proceso, de manera contemporánea, esta teoría moral se ha transformado en una cuestión de individualidad, de lo que se aprueba o reprueba, basado grandemente en una carga emotiva de las situaciones. Esta teoría de emotividad no es aceptada por las teorías filosóficas contemporáneas; sin embargo, al no ser aceptada por la filosofía, es irónicamente muy aceptada por el resto de la población, enraizada en la cultura (Grosh). Lo anterior produce el comportamiento posmoderno en el que ya no se identifica un discurso moral cohesivo, un discurso único, sino todo se vuelve relativo y dependiente de la razón del individuo. Así, el concepto posmoderno de espiritualidad se vuelve muy genérico y anti-filosófico.

Según Grosh, la división del concepto de espiritualidad sucedió cuando la teología se separa de la filosofía. Según Hadot en Grosh (p. 235), el filosofo Suarez en su trabajo Metaphysicarum disputationum Tomi Duo hace énfasis de la exclusión mutua de los conceptos teología y filosofía, y así, el concepto de espiritualidad queda diseccionada de la filosofía, para pertenecer a la teología. Es por eso la problemática actual sobre la pertenencia del concepto espiritualidad, y por lo tanto, las prácticas espirituales se clasifican como prácticas religiosas. Sin embargo, Grosh indica que estas prácticas espirituales pueden tener un rastreo filosófico, pero deben identificarse como las que ocurrieron antes de esta bifurcación. Para tal identificación, se pueden considerar los trabajos de Platón, y especialmente la Ética Nicomaquea de Aristóteles, estoicos y Plotino

La problemática principal en este tiempo posmoderno es que el concepto de espiritualidad ha perdido ese sentido de pertenencia a una tradición en particular, de coherencia interna, de una referencia histórica, por lo tanto, se convierte en un matizado de colores en el que no se distingue un patón en sí mismo. No es enteramente negativo, según Grosh, ya que el individuo ha logrado encontrar un beneficio de la falta de un patrón al interiorizarlo de manera individual y personal, y así la espiritualidad se convierte en un concepto aplicable a todos los momentos, bajo todos los matices, con múltiples críticas ya que no posee fundamentos solidos al no estar enraizado en ninguna tradición en particular. Esto produce la volatilidad del concepto, y su poca estima en nuestros tiempos.

La palabra espiritualidad en su concepto filosófico original, iniciando con Platón se divide en dos términos: Psyche y Pneuma. Para Platón, psyche se conectaba con el concepto del alma inmortal, que

2 Francisco de Suarez, 1597, Metaphysicarum Disputationum Tomi duo. (Salamanca); fuente : F. Copleston, 1953, A History of Philosophy Vol.lll: Ockham to Suarez. (Londres : Search Press); pp 353-405; pp 445-7 
provenía de una fuerza del universo, y así, el alma trascendería al cuerpo. Por el contrario, los estoicos no consideraban la inmortalidad del alma, entonces ellos aportan el término pneuma, o aliento de vida, y así, el enfoque se transforma en materialista al ser el cuerpo el contenedor de la vida y del alma que vive según el cuerpo. Esta definición no clarifica lo que significa ser humano. Entonces, la manifestación de la espiritualidad trasciende a un plano diferente, o místico. En 1922, Wittgenstein explica que existen cosas que no pueden ser traducidas en palabras, simplemente se vuelven manifiestas, y estas son las que son místicas.

De esta manera, Grosh introduce su concepto de espiritualidad como el acto de vivir una vida de acuerdo con un propósito interno, confeccionado por el individuo mismo, en el cual se reconoce el tan vasto, complejo y místico aspecto de lo que significa ser humano, quien comparte un espacio con otro ser humano que también tiene esta percepción individual, y ambos comparten el extraño y maravilloso regalo de estar vivos.

De esta manera, se concluye que la espiritualidad debe ser un proceso de aprendizaje, centrado en el propósito de la vida del individuo. Según Aristóteles, el propósito se conecta con la práctica de las virtudes morales, virtudes intelectuales, y educación como hábitos, lo cual produce una vida que merece ser vivida, la cual obtiene los frutos de la felicidad. Según Grosh, se pueden definir ejercicios prácticos para experimentar la espiritualidad según las cuatro escuelas clásicas principales: platonismo, aristotelismo, estoicismo, epicureísmo. Tales escuelas proporcionan lo que se conoce como una 'dieta espiritual' compilada por Hadot (1995) para cultivar las cualidades de la mente y el carácter necesarias para alcanzar nuestro propósito. Estas cualidades son: la investigación, la investigación profunda, lectura, la habilidad de escuchar, el enfoque, la maestría individual, las terapias de las pasiones, el recuerdo de las cosas buenas, y la completitud de las tareas o actividades. ${ }^{3}$ Estos ejercicios griegos forman parte de la práctica espiritual que nos ayudará a alcanzar nuestro propósito. Como crítica, Grosh hace explícito que las prácticas cristianas son fundamentalmente morales, y este modelo moral ha sido practicado a través de la imitación de ejemplos filosóficos griegos.

Werner Jaeger (p. 174) argumenta que los poemas de La Iliada y La Odisea fueron escritos para educar sobre las virtudes necesarias para mantener una buena vida. Entonces el propósito de la literatura y la poesía es la educación del individuo. Para esto, es necesario es-

3 "Zetesis, skepsis, anagnosis, akroasis, prosoché, enkrateia, meletai"- La cita proviene de P. Hadot, 1995, Philosophy as a Way of Life: Spiritual Exercises from Socrates to Foucault. (ed. A. Davidson; trans. M. Chase. Oxford: Blackwell), p. 84. 
tablecer bajo qué propósito al observar los confines de la historia en sí misma, y hacer una lectura que nos permita acercarnos a esa enseñanza final, no únicamente por el disfrute de la literatura, sino con el propósito que el autor entendía como primordial al momento de desarrollar su historia.

Lo anterior es importante para evitar la noción moderna de que la espiritualidad trasciende culturas y no debe ser dogmática, lo cual es un error. Cada religión y cada tradición tiene su agrupación de teorías y prácticas que son importantes en sí mismas. Se recomienda, entonces, un acercamiento muy cuidadoso de manera exegética y hermenéutica para no caer en la cuestión de un imperialismo cultural, y cometer el error de sacar la tradición de su contexto cultural, filosófico, histórico, narrativo, estructural y de significado y propósito.

\section{Características de la Literatura Medieval}

La literatura inglesa medieval incluye elementos temáticos recurrentes que establecen una homogeneidad en los relatos. La ausencia de amor romántico sin eliminar la presencia de una dama inalcanzable es importante en la trama. Ésta ausencia responde a la búsqueda principal de próposito en el héroe. También es elemento importante la recreación del mundo con el uso de metonimia, metáfora o alegorías. Esta descripción viva se corona de elementos en ocasiones supernaturales que podrían representar la tradicion oral anglosajona de tono elegiaco. Los elementos técnicos del verso tenían el proposito de ayudar a la recitación oral dentro de los grandes salones de los guerreros anglosajones. Presentan un patrón aliterativo con una pausa o cesura a la mitad del verso que emularia el ritmo del inglés antiguo. Sin embargo, en general, no existe un patrón rítmico adicional al creado por la aliteración. Para describir objetos o ideas se usan los keningares o palabras compuestas para, como una forma arcaica de la metáfora.

La épica anglosajona representa el comportamiento ideal del guerrero. Indica de qué manera se percibía, y de qué manera debía ser. El guerrero héroe debía establecer un ejemplo a seguir como luchador incansable, domador de caballos y a veces dragones, líder en el campo de batalla, y también capaz de cultivar la tierra y cantar andanzas de otros héroes. Sin embargo, la verdadera marca del héroe, y su camino a la realeza, era su generosidad. Solo el rey podría ser capaz de ofrecer, y los que recibían le recompensarían con su lealtad. Todos estos elementos están presentes en la poesía que se encuentra en el Códice de Exeter y el relato épico de Beowulf incluido en el Manuscrito Cotton Vitellius. 
El texto inglés medieval presenta múltiples complicaciones al momento de su estudio. En este contexto, el texto está inicialmente escrito en una lengua muerta que ha sido difícil para los traductores ya que algunas páginas de los manuscritos se encuentran dañadas, incompletas, o inexistentes.

Adicionalmente, la historia marco tiene su origen en leyendas y tradición oral que fue popular hasta antes del siglo VII. Sin embargo, existen pocos registros, y estudiosos que estén capacitados para transmitirlos en castellano.

El caso del poema épico de Beowulf es consistente con estos retos. El registro en Códice Nowell (Cotton Vitellius) se rastrea al siglo XI. El poema, sin embargo, es considerado el más antiguo ejemplo de literatura anglo-sajona, posiblemente fijado al texto entre el $521 \mathrm{y}$ 1026. Es un poema épico escrito en inglés antiguo o anglo-sajón, sin rima, pero siguiendo la estructura aliterativa del verso germano, en el que se narra la vida del guerrero Beowulf y sus enfrentamientos con 2 monstruos y un dragón.

La dificultad del análisis de un texto de esta naturaleza versa en el entendimiento del propósito original, el cual es elusivo para nosotros. No existe certeza de la autoría, haya sido individual o colectiva, tampoco se define si el texto sufrió modificaciones como otros poemas elegíacos transcritos por monjes cristianos.

Debido a estas complicaciones, múltiples estudios sobre el propósito del texto se han desarrollado a la luz de explicaciones naturalistas, cristianas, de vasallaje, o intentos históricos de reconstrucción de la cultura germana. Básicamente, poco se puede afirmar del poema de Beowulf. Yo intentaré retomar elementos concretos que se incluyen principalmente en Beowulf, pero que son representativos del imaginario de la época medieval. Para lograr esto, se analizarán otros ejemplos de literatura de la época incluidos también en este artículo.

Un ejemplo de esto es identificar el elemento del bosque y su implicación en la narrativa del poema. Históricamente, se puede verificar la alienación de grupos considerados paganos durante la ocupación romana en el año 55 A.C. Estos grupos, en su mayoría celtas, fueron empujados fuera del área controlable por el imperio romano, y por lo tanto, se establecieron en áreas boscosas completamente antagonistas a la civilidad traída por los romanos. Considero entonces que el bosque mantiene una muy notable importancia no solo en la narrativa de las leyendas y poemas de tales grupos, sino en la definición del concepto de Espiritualidad que quisiera abordar.

La naturaleza de la Elegía nos invita también a explorar el deseo del narrador, e invariablemente, los encuadres de la lirica, posiblemente agregados por monjes, le insertan la espiritualidad religiosa 
a la obra. El cristianismo y el comportamiento Anglosajón no eran antagonistas. Los Anglosajones buscaban imitar a los héroes, y los cristianos buscaban imitar a Cristo, lo cual ofrece una perspectiva de heroísmo muy interesante ya que la literatura buscaba instruir. Entender la naturaleza de la instrucción será fundamental.

Sin embargo, como se describe en la siguiente cita: "la explicación consiste en quitar toda extrañeza al objeto que se examina de modo que se lo vuelva más familiar... [entonces] estos objetos deben inscribir a nuestro contexto para adquirir sentido."

Los textos seleccionados son representativos de la tradición anglosajona. El texto de Beowulf, historia de un guerrero de la tierra de los geatas que decide ir a la tierra de los daneses para vencer a un monstruo que ha estado atormentando el trono y el centro de reunion del reino danés. Este monstruo llamado Grendel deciende de Caín, como se creía tradicionalmente durante los primeros siglos. Caín fue el primer asesino al tomar la vida de su hermano Abel, por lo tanto, fue condenado a vagar sin pertenecer nunca a ningún pueblo, y con la amenaza de morir de la misma manera en la que había quitado la vida de Abel. Para el pensamiento medieval, cualquier discapacidad, o malperfecto simbolizaría una corrupción de espíritu, posiblemente no del doliente, sino de generaciones anteriores, porque como se inscribe en GENESIS, las consecuencias del pecado se visitan hasta la tercera o cuarta generación. Esta línea de descendencia es obsena, marcada por elementos deformes debido al pecado de Cáin. La referencia es principalmente cristiana.

El cristianismo se había establecido en Inglaterra desde el siglo IV. Sin embargo, el sincretismo se dio de manera lenta. Los siguientes textos son de naturaleza elegiaca. Uno de los ejemplos es el poema The Seafarer, o el Navegante, que narra la historia de un exiliado, con un cierto tono alegre ya que para el navegante, la vida en la soledad del altamar es benéfica para su espíritu deseoso de encontrar honra de nuevo, perspectiva que ataca el liderazgo juvenil e inmaduro del momento.

Bede, un monje cuyo estilo es similar al estilo biblico, es entregado al monasterio desde muy temprana edad, lugar en donde aprende todo lo que expresa en sus escritos. Gracias a Bede, tenemos un eslabon al mundo anglosajon ya que él es el responsable de la compilación de la historia anglosajona a manera de un religioso observando el desarrollo histórico de una nación. Se puede criticar la falta de objetividad de su estilo, ya que es visiblemente inclinada a una perspectiva religiosa. Lo anterior forma el común denominador en la escritura de la época. Bede el venerable hace un recuento de los momentos prin- 
cipales de la historia inglesa y los documenta en su The Ecclesiastical History of the English Church and People. Las selecciones se asemejan a la parabolas expresadas por Jesús ya que invariablemente buscan una transformación espíritual, en este caso dogmática, en el oyente.

Las baladas son canciones repetidas de generación a generación. Fueron durante siglos de naturaleza oral, posiblemente desde el siglo IX hasta el siglo XVIII cuando se fijaron en texto

\section{Conclusión}

Los británicos, después de haber sido expuestos a estos cambios culturales y literarios, estaban dispuestos a cruzar fronteras, conocer otros lugares con culturas distintas. Este es el propósito fundamental de la literatura. Y lograr hacer la literatura accesible es imprescindible para cualquier ser humano. Algunos sistemas educativos ya consideran la implementación de materias que exploren el desarrollo en torno a la espiritualidad, ya que se asociaría a un desarrollo integral del alumno.

Algunos críticos indican que una obra de arte no se vincula a su contexto histórico, por lo tanto, explicar la obra es un intento de explicar el cómo se compuso la obra y el contexto en el que existe, la exégesis, sin embargo, nosotros contendemos con esa idea.

La espiritualidad entendida como una agrupación de cualidades que se supeditan a la existencia humana. Esto es, que no están determinadas por la mortalidad, y por lo tanto, se vuelven elementos trascendentales del ser humano que se han representado en la literatura a través de los tiempo. Mi enfoque será sobre el texto inglés medieval.

El periodo indicado como enfoque de estudio sigue la línea de textos que son propios de la epoca anglosajona. Momento histórico en el que se está gestando una adaptación cultural masiva entre las tribus invasoras, los remanentes de la isla, y los representates eclesiasticos como misioneros. Esto produce una amalgama de creencias fundamentalmente paganas con miras a la transformación cristiana. Esta transformación no se dio de la noche a la mañana, y comunmente, el proceso de conversión sucedía de arriba abajo, del rey a los campesinos. Así, la dificultad de una correcta implementación, principalmente debido a la falta de educación por parte de los comunes, permitía creaciones literarias, provenientes de una oralidad, en las que los rasgos paganos no podían ser eliminados. Este periodo anglosajón termina con el inicio de la leyenda artúrica, la cual no exploraremos en este momento. 
Los textos están escritos en inglés antiguo o anglosajón, variante de una lengua germanica arcaica; siendo una lengua muerta, se tiene poca información. Estas culturas fundamentaban su tradición en la oralidad, haciendo de la fijación de la selección al manuscrito un proceso elusivo. Se desconocen la mayoría de los autores de estos textos medievales, y los manuscritos que los contienen han sido severamente maltratados; no obstante, continuan siendo la única fuente sobre los procesos socio-culturales y literarios de la época anglosajona.

El elemento problemático yace sobre la necesidad de indicar la fecha de fijación de la selección al texto para mayor comprensión de estos eventos histórico-sociales. Cuando la selección existe en la tradicción oral, la componen elementos transformativos que se desarrollan de generación a generación. Una vez fijado, el texto se transforma en imperecedero, inamovible, pero distante especialmente en situaciones en la que ninguna otra referencia cultural se ha encontrado. Distante también por una lengua poco accesible, lo que provoca una variedad de acercamientos de traducción. Múltiples versiones que no pueden traspasar los linderos de las mismas palabras, y así, se quedan cortas. Esta traducción se inscribe entonces en otro momento histórico, bajo parametros diferentes a aquellos en los que la selección fue concebida. Fijados en un siglo nuevo, llevan también consigo la carga ideológica del momento. El traductor no puede eliminar su ideología, en ocasiones inscrita como subtexto y observada en la recepción del texto.

La filosofía se ha interesado en el estudio del lenguaje, por lo tanto, la manera en la que se comunican las ideas a través de los diferentes instrumentos con los que contamos, ya sea el discurso real o literario, se establece como aspecto central del estudio de la filosofía. Por lo tanto, estudiar la espiritualidad conlleva de manera inherente un enfoque filosófico; la manera en la que se entiende la espiritualidad es inherentemente un problema filosófico. 


\section{REFERENCIAS}

Astin, Alexander W. "Why Spirituality Deserves a Central Place in Liberal Education." En Liberal Education, 2004: 34-41.

Grosh, Paul. "Paideia: philosophy educating humanity through spirituality". En International journal of children's spirituality, 2000: 229-237.

McNamara, John. Translation of Beowulf. New York: Barnes \& Noble Books, 2005.

Matus, Carolina Valenzuela. "Espiritualidad Medieval y Antigüedad Clásica. La Historia Eclesiástica Indiana de Fray Jeronimo de Mendieta”. En Revista Electrónica Historias del Orbis Terrarum, 2014: 157-175.

Suárez, Francisco. Metaphysicarum disputationum Tomi Duo. Salamanca: Salmanticae : apud Ioannem \& Andream Renaut fratres, 1597.

Tolkien, J.R.R. Beowulf, a Translation and Commentary. Great Britain: Harper Collins Publishers, 2014.

Fraile, Guillermo. Historia de la Filosofía. Biblioteca de Autores Cristianos, 1997.

Meletinsky, Eleazar. "Sociedades, Culturas y Hecho Literario.” Teoría Literaria, 2002, pp. 17-35.

Montero, Miquel Aguilar. "Fundamentos teóricos de la Épica Universal en la literatura germánica altomedieval : El Poema de Beowulf." Espéculo. Revista de Estudios Literarios, 2009.

Schönecker, Dieter, and Elke Elisabath Schmidt. "Kant's Ground-Thesis. On Dignity and Value in the Ground Work". En Journal of Value Inquiry, vol. 52, no. 1, 2018, pp. 81-95, doi:10.1007/s10790-0179603-z.

Wевв, Jon R., et al. "Ritualistic, Theistic, and Existential Spirituality: Initial Psychometric Qualities of the RiTE Measure of Spirituality". En Journal of Religion and Health, vol. 53, no. 4, 2014, pp. 972-85, doi:10.1007/s10943-013-9697-y.

Este artículo se publica bajo una licencia de Creative Commons Reconocimiento-NoComercial 4.0 Internacional, y puede ser usados gratuitamente para fines no comerciales, dando los créditos a los autores y a la revista. 